\title{
INCREASED VIRAL TITERS AND SUBTLE CHANGES IN PLAQUE MORPHOLOGY UPON PASSAGE OF SARS-COV IN CELLS FROM DIFFERENT SPECIES
}

\author{
Laura Gillim-Ross, Lindsay K. Heller, Emily R. Olivieri, \\ and David E. Wentworth*
}

\section{INTRODUCTION}

Despite rapid advances in our knowledge of SARS-Coronavirus (SARS-CoV), the regions of the virus spike glycoprotein and host receptor that are important for virus entry remain to be completely elucidated. The tropism of SARS-CoV for cells derived from many diverse species was analyzed by virus titration and by the use of a multiplex RTPCR assay that differentiates input virus from virus that has entered a cell and initiated replication. ${ }^{1}$ Cells derived from monkey, human, and mink were shown to be productively infected by SARS-CoV. Interestingly, the level of virus produced varied dramatically $\left(4 \log _{10}\right)$ between the susceptible cell lines. Human angiotensin-converting enzyme 2 (ACE2) was shown to have SARS-CoV receptor activity. ${ }^{2}$ Using conserved oligonucleotide primers and RT-PCR to amplify ACE2, we determined that the SARSCoV susceptible cells expressed ACE2 RNA. We hypothesized that passage of SARS$\mathrm{CoV}$ in cell lines expressing different levels of ACE2, or ACE2 from novel species (e.g., mink), will lead to mutations in the spike glycoprotein gene, and/or in other genes that enhance virus replication. SARS-CoV/Urbani was passaged in susceptible cell lines derived from monkey, human, and mink. The titer of SARS-CoV in the supernatants increased upon passage, and viral plaques in VeroE6 cells showed subtle differences from wild-type SARS-CoV/Urbani. We are currently analyzing the sequence of the SARS-CoV quasi-species selected by passage; such analysis may identify regions of the spike protein that are critical for virus binding and/or fusion. The identification of regions within the viral spike glycoprotein critical for interaction with the receptor is important for the development of antivirals and/or immunogens for vaccine development.

\footnotetext{
* Laura Gillim-Ross, Lindsay Heller, New York State Department of Health, Albany, New York 12002. Emily Olivieri, David E. Wentworth, New York State Department of Health and State University of New York, Albany, New York 12002.
} 


\subsection{Background}

In 2002-2003, an outbreak of severe acute respiratory syndrome (SARS) spread from Southern China to 28 other countries. ${ }^{3}$ SARS infected at least 8,096 people, and led to 774 deaths. $^{4}$ A novel coronavirus (CoV), SARS-Coronavirus (SARS-CoV) was identified as the causative agent of the outbreak. ${ }^{5-7}$ Although the origin/reservoir of SARS-CoV has not been identified, the identification of SARS-CoV-like viruses and anti-SARS-CoV antibodies, in several species in live animal markets and on farms in China, strongly suggests that the SARS outbreak resulted from zoonotic transmission(s). ${ }^{8}$, ${ }^{9}$ Specifically, SARS-CoV-like viruses and antibodies to SARS-CoV have been detected in Himalayan palm civets (Paguma larvata). ${ }^{8,9}$ Rapid evolution of the SARS-CoV in palm civets and humans ${ }^{10}$ suggests that the predecessor of SARS-CoV is endemic in another common source, and that palm civets probably played a role as an intermediate or amplifying host, leading to zoonosis.

CoVs belong to the order Nidovirales, family Coronaviridae. CoVs are a diverse group of enveloped, positive-sense RNA viruses. The CoV genome, 27-32 kilobases in length, is the largest of the known RNA viruses. CoV attachment, and fusion of the viral lipid envelope with host lipid membrane, is mediated by trimeric spike (S) glycoproteins that project from the virion. ${ }^{11}$ Although CoVs infect a wide variety of species, including dogs, cats, cattle, mice, birds, and humans, the natural host range of each strain is typically limited to a single species. ${ }^{11}$ Interaction of virus $\mathrm{S}$ with host cell receptors is a major determinant of the species specificity and tissue tropism of CoVs. Upon entry into target cells, CoVs initiate viral replication, utilizing a complex discontinuous RNA transcription mechanism to generate 3' co-terminal subgenomic RNAs, which share a short 5 ' leader sequence. ${ }^{12}$

Using a multiplex RT-PCR assay that detects SARS-CoV genomic and subgenomic RNA, we previously showed that cells derived from African green monkey kidney (VeroE6), human liver (Huh7), human kidney (HEK-293T), and mink lung (Mv1Lu) were all productively infected by SARS-CoV. ${ }^{1}$ The titer of virus produced varied dramatically ( $4 \log _{10}$ ) between the different susceptible cell lines. ${ }^{1}$ At $24 \mathrm{~h}$ post-inoculation, VeroE6 cells released the highest titer of virus $\left(5 \times 10^{7} \mathrm{TCID}_{50} / \mathrm{ml}\right)$, Huh7 cells $\left(2 \times 10^{4} \mathrm{TCID}_{50} / \mathrm{ml}\right)$, HEK-293T $\left(5 \times 10^{3} \mathrm{TCID}_{50} / \mathrm{ml}\right)$, and Mv1Lu $\left(8 \times 10^{2} \mathrm{TCID}_{50} / \mathrm{ml}\right)$ released much lower titers of virus. ${ }^{1}$

We hypothesized that passage of SARS-CoV in cells with varying levels of receptor, or with species-specific differences in the receptors, will select for SARS-CoV with mutations in the $\mathrm{S}$ gene, and/or in other genes, that are advantageous to virus replication. Therefore, analysis of selected mutants will identify regions of $S$ that are critical to entry of the SARS-CoV. In this study, we generated mutant SARS-CoVs by subjecting SARS$\mathrm{CoV} /$ Urbani to selective pressure, primarily at the level of host cell receptors. Virus was serially passaged 12 times in cell lines that were derived from African green monkey, human, and mink. In addition, two human cell lines that express differing levels of receptor were used. To determine whether phenotypic changes had been selected, we analyzed the amount of virus released into the supernatant, as well as the plaque morphology of SARS-CoV variants, after passage in each of the cell lines. 


\section{RESULTS AND DISCUSSION}

Human ACE2 and CD209L were recently identified as functional receptors for SARS-CoV. ${ }^{2,13}$ Although both are functional receptors, human ACE2 is a more efficient receptor for SARS-CoV than is CD209L. The cell lines that we identified as productively infected by SARS-CoV were assayed for the presence of ACE2. One-step RT-PCR (Qiagen) with oligonucleotide primers conserved between mouse and human ACE2 was used to amplify ACE2 RNA from $1 \mu \mathrm{g}$ of total RNA in susceptible cells derived from various species. Sense primer (ACE2-902, 5'-CTTGGTGATATGTGGGGTAGA) and an antisense primer (ACE2-1548R, 5'-CGCTTCATCTCCCACCACTT) amplify a 646-base-pair fragment of ACE2 when RNA is expressed. SARS-CoV susceptible Huh7, VeroE6, HEK-293T, and Mv1Lu cells ${ }^{1}$ were assayed for ACE2 transcript. ACE2 RNA was detected in all of the susceptible cell lines (Figure 1A). Qualitatively, the level of ACE2 differed among the various cell lines. VeroE6 had the strongest ACE2 amplicon, whereas Mv1Lu cells showed the weakest product (Figure 1A). Although ACE2 amplicons from Mv1Lu cells are weak, RT-PCR amplification with mink-specific primers generates a robust amplicon (Heller et al., this volume). The level of protein expressed among these four cell lines was greatest in VeroE6, and lowest in HEK-293T (Olivieri and Wentworth, unpublished data). Of the cell lines susceptible to SARS-CoV, Mv1Lu cells, which were derived from mink lung, are most closely related to those of the Himalayan palm civet. The predicted amino acid sequence of mink ACE2 has $83 \%$ identity with the human ACE2 sequence and $88 \%$ identity with palm civet ACE2 (Heller et al., this volume). Therefore, we analyzed the ability of mink ACE2 to function as a SARS-CoV receptor. Expression of mink ACE2 in normally non-permissive BHK-21 cells resulted in SARS-CoV infection (Heller et al., this volume). Taken together, the data show that these four cell lines all express ACE2 that is a functional receptor for SARS-CoV.

Variation in the levels of virus production among the four cell lines may correspond to differing levels of ACE2 expression, or to species-specific variations in ACE2. VeroE6 cells produce high titers of SARS-CoV, ${ }^{1}$ and they also express high levels of ACE2. ${ }^{2}$ In contrast, HEK-293T cells produce significantly less SARS-CoV (4 $\log _{10}$ lower titer), ${ }^{1}$ and they express very low levels of ACE2. ${ }^{2}$ SARS-CoV/Urbani (previously passaged 4 times in VeroE6) was passaged in VeroE6, Huh7, HEK-293T, or Mv1Lu cells for a total of 12 passages. Two flasks of each cell line were inoculated with SARS-CoV/Urbani (Pass 4) at an MOI of 1.0 (replicates A and B). Cells were observed every $24 \mathrm{~h}$ for cytopathic effect, and viral supernatants were collected at $24 \mathrm{~h}, 48 \mathrm{~h}$, or $72 \mathrm{~h}$ postinoculation. The viral supernatants were either immediately passed, or were titered in VeroE6 cells before subsequent passage. Cytopathic effect was observed only in VeroE6 cells throughout the experiment. Viral titers were determined for all passages by TCID $_{50}$ in VeroE6 cells, as previously described. ${ }^{1}$ The titer of virus produced by VeroE6 cells showed a modest increase after passage (Figure 1B). In contrast, virus passaged in Huh7 cells increased in titer by $\sim 2.5 \log _{10}$ (Figure 1B). Passage of virus in HEK-293T cells resulted in a decrease in virus titer from $8 \times 10^{6} \mathrm{TCID}_{50} / \mathrm{ml}$ to $2 \times 10^{5} \mathrm{TCID}_{50} / \mathrm{ml}$ (Figure $1 \mathrm{~B}$ ). The replicates (A and B) of VeroE6, Huh7, and HEK-293T passage had very similar titers. In contrast, one of the replicates (B) of Mv1Lu passage showed a dramatic increase in viral titer upon passage. The titer increased from $1 \times 10^{3} \mathrm{TCID}_{50} / \mathrm{ml}$ (average of passages 1 and 2 ) to $3.7 \times 10^{7} \mathrm{TCID}_{50} / \mathrm{ml}$ (average of passages 11 and 12); the latter titer is similar to that produced by VeroE6 cells (Figure 1B). The other replicate Mv1Lu passage (A) never reached a titer exceeding $2 \times 10^{2}$ $\mathrm{TCID}_{50} / \mathrm{ml}$, and was not detectable by passage 7 (data not shown). Thus, the titer of replicate 
A.

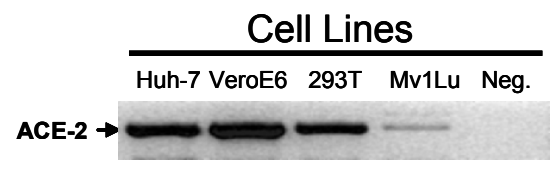

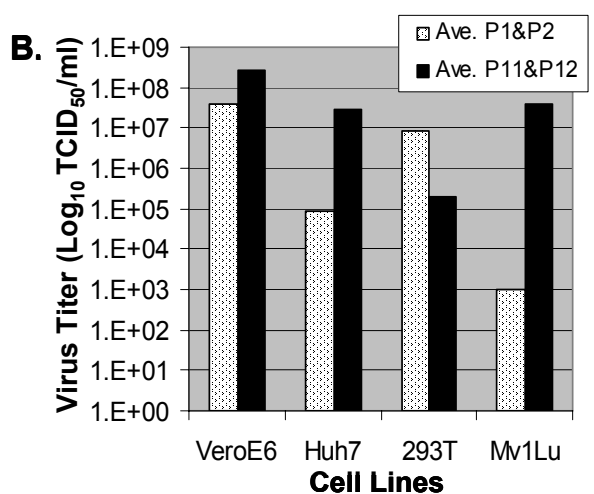

Figure 1. A. ACE2 RNA expression in cell lines susceptible to SARS-CoV. Negative image of ethidium bromide stained gel of RT-PCR amplicons from $1 \mu \mathrm{g}$ of total RNA. Water was used as template for negative control reaction (Neg.). B. Passage of SARS-CoV in different cell lines results in changes in viral titer. SARS$\mathrm{CoV} /$ Urbani was passaged 12 times in African green monkey kidney (VeroE6), human liver (Huh7), human kidney (293T), or mink lung (Mv1Lu) cell lines. The average of viral titer of passage 1 and 2 (P1 \& P2) was compared with average titer of passage 11 and 12 (P11 \& P12).

A decreased until the virus could not be sustained under the passage conditions. Alternatively, adaptation to mink ACE2 results in poor interaction with African green monkey ACE2, and consequently an artificially low $\mathrm{TCID}_{50}$ in VeroE6 cells.

To determine whether the observed changes in viral titer correlated with changes in plaque phenotype, we analyzed viral supernatants obtained from the four cell lines at passage 12 (P12). We compared the plaque phenotype of the passaged variants with the parental wild-type virus (Urbani) using VeroE6 cell monolayers. VeroE6-P12 virus did not exhibit a significant change in plaque morphology when compared to Urbani. Both Urbani and VeroE6-P12 contained a population of subtly pleomorphic plaques. The average Urbani plaque was $0.7 \mathrm{~mm}$, and the average VeroE6-P12 plaque was $1.0 \mathrm{~mm}$ in diameter. Huh7-P12, Mv1Lu-P12, and HEK-293T-P12 variants all showed increases in average plaque diameter to $1.2,1.5$, and $1.8 \mathrm{~mm}$, respectively.

Changes observed in virus titer and in plaque morphology after 12 passages show that viral adaptation occurred as a result of the various selective pressures exerted by growth in primate, human, or mink cells. Species-specific differences in ACE2 expressed by these cells likely played a strong role in the selection process. In addition, differences in the level of human ACE2 expressed by Huh7 and HEK-293T likely selected for viruses with S glycoproteins that differ in their affinities for human ACE2, or that have enhanced abilities to use alternative receptors. For example, the largest increase in plaque size occurred after selection in HEK-293T cells, which express the lowest amount of ACE2, among the cell lines studied. We are currently analyzing the consensus nucleotide sequences of the quasi-species of the passaged viruses, to identify potential changes in $\mathrm{S}$ and/or other portions of the genome. We are also analyzing plaque-purified variants, because the plaque morphologies obtained suggest multiple genotypes exist in the passaged population of viruses. Comparison of the amino-acid sequence differences between human and mink ACE2 in the S binding domain, coupled with analysis of the adaptive changes occurring within $\mathrm{S}$, will provide a better understanding of the SARSCoV S-ACE2 interaction. Additionally, selection of SARS-CoV S variants that bind 
human ACE2 with higher affinity than does wild-type SARS-CoV will aid in the development of inhibitors that block viral entry. Lastly, the identification of changes in SARS-CoV that alter the tropism of the virus will provide additional information as to the mechanism(s) of zoonotic transmission of SARS-CoV.

\section{ACKNOWLEDGMENTS}

We thank Drs. William Bellini and Thomas Ksiazek for providing SARS$\mathrm{CoV} /$ Urbani, and Dr. Aleem Siddiqui for providing Huh7 cells. The authors thank Noel Espina for his expert assistance, the Wadsworth Center Molecular Genetics Core for DNA sequencing, and the Wadsworth Center Tissue Culture Core Facility. L.G.-R. was supported by an appointment to the Emerging Infectious Diseases Fellowship program administered by the Association for Public Health Laboratories and funded by the CDC, and E.O. was supported by NIH/NIAID training grant T32AI05542901A1. This study was also funded in part by the Public Health Preparedness and Response to Bioterrorism cooperative agreement between the Department of Health and Human Services, and the Centers for Disease Control and Prevention, a research agreement with Diagnostic Hybrids Inc., and NIH/NIAID grants N01-AI-25490, and P01-AI-0595760.

\section{REFERENCES}

1. L. Gillim-Ross, J. Taylor, D. R. Scholl, J. Ridenour, P. S. Masters, and D. E. Wentworth, Discovery of novel human and animal cells infected by the severe acute respiratory syndrome coronavirus by replicationspecific multiplex reverse transcription-PCR, J. Clin. Microbiol. 42, 3196-3206 (2004).

2. W. Li, M. J. Moore, N. Vasilieva, et al., Angiotensin-converting enzyme 2 is a functional receptor for the SARS coronavirus, Nature 426, 450-454 (2003).

3. N. S. Zhong and G. Q. Zeng, Our strategies for fighting Severe Acute Respiratory Syndrome (SARS), Am. J. Respir. Crit. Care Med. 168, 7-9 (2003).

4. Summary of probable SARS cases with onset of illness from 1 November 2002 to 31 July 2003, World Health Organization, http://www.who.int/csr/sars/country/table2004_04_21/en/index.html, 4-21-2004.

5. C. Drosten, S. Gunther, W. Preiser, et al., Identification of a novel coronavirus in patients with severe acute respiratory syndrome, N. Engl. J. Med. 348, 1967-1976 (2003).

6. T. G. Ksiazek, D. Erdman, C. S. Goldsmith, et al., A novel coronavirus associated with severe acute respiratory syndrome, N. Engl. J. Med. 348, 1953-1966 (2003).

7. J. S. Peiris, S. T. Lai, L. L. Poon, et al., Coronavirus as a possible cause of severe acute respiratory syndrome, Lancet 361, 1319-1325 (2003).

8. Y. Guan, B. J. Zheng, Y. Q. He, et al., Isolation and characterization of viruses related to the SARS coronavirus from animals in southern China, Science 302, 276-278 (2003).

9. C. Tu, G. Crameri, X. Kong, et al., Antibodies to SARS coronavirus in civets, Emerg. Infect. Dis. 10, $2244-$ 2248 (2004).

10. H. D. Song, C. C. Tu, G. W. Zhang, et al., Cross-host evolution of severe acute respiratory syndrome coronavirus in palm civet and human, Proc. Natl. Acad. Sci. USA 102, 2430-2435 (2005).

11. K. V. Holmes, in: Fields Virology, Vol. 1, edited by D. M. Knipe, P. M. Howley, D. E. Griffin, R. A. Lamb, M. A. Martin, and B. Roizman (Lippincott Williams \& Wilkins, Philadelphia, 2001), pp. 1187-1203.

12. S. G. Sawicki and D. L. Sawicki, Coronavirus transcription: a perspective, Curr. Top. Microbiol. Immunol. 287, 31-55 (2005).

13. S. A. Jeffers, S. M. Tusell, L. Gillim-Ross, et al., CD209L (L-SIGN) is a receptor for severe acute respiratory syndrome coronavirus, Proc. Natl. Acad. Sci. USA 101, 15748-15753 (2004). 